\title{
Correction to: The CAPOS mutation in ATP1A3 alters Na/K-ATPase function and results in auditory neuropathy which has implications for management
}

\author{
Lisbeth Tranebjærg ${ }^{1,2,3}$. Nicola Strenzke ${ }^{4}$ Sture Lindholm ${ }^{5}$. Nanna D. Rendtorff ${ }^{2} \cdot$ Hanne Poulsen $^{6}$. \\ Himanshu Khandelia ${ }^{7}$. Wojciech Kopec ${ }^{7,8}$. Troels J. Brünnich Lyngbye ${ }^{9}$. Christian Hamel ${ }^{10,11,12}$. Cecile Delettre ${ }^{11,12}$. \\ Beatrice Bocquet ${ }^{10,11,12} \cdot$ Michael Bille $^{13} \cdot$ Hanne H. Owen ${ }^{14} \cdot$ Toke Bek $^{15} \cdot$ Hanne Jensen $^{16} \cdot$ Karen $_{\text {stergaard }}{ }^{17}$. \\ Claes Möller ${ }^{18}$. Linda Luxon ${ }^{19}$. Lucinda Carr ${ }^{20}$. Louise Wilson ${ }^{21} \cdot$ Kaukab Rajput $^{22}$. Tony Sirimanna ${ }^{23}$. \\ Katherine Harrop-Griffiths ${ }^{24}$. Shamima Rahman ${ }^{25}$. Barbara Vona ${ }^{26}$. Julia Doll ${ }^{26}$. Thomas Haaf ${ }^{26}$. Oliver Bartsch ${ }^{27}$. \\ Hendrik Rosewich ${ }^{28}$. Tobias Moser ${ }^{29} \cdot$ Maria Bitner-Glindzicz ${ }^{21,25}$
}

Published online: 12 February 2018

(c) Springer-Verlag GmbH Germany, part of Springer Nature 2018

\section{Correction to: Human Genetics} https://doi.org/10.1007/s00439-017-1862-z

The following information was inadvertently omitted in the original publication:

TM and NS were supported by the German Research Foundation through the Collaborative Research Center 889.

The original article can be found online at https://doi.org/10.1007/ s00439-017-1862-z.

Lisbeth Tranebjærg

tranebjaerg@ sund.ku.dk

$\bowtie$ Maria Bitner-Glindzicz maria.bitner@ucl.ac.uk

1 Department of Otorhinolaryngology, Head and Neck Surgery and Audiology, Rigshospitalet/Bispebjerg, Copenhagen, Denmark

2 Department of Clinical Genetics, The Kennedy Center, Copenhagen University Hospital, Copenhagen, Denmark

3 Institute of Clinical Medicine, University of Copenhagen, Copenhagen, Denmark

4 Auditory Systems Physiology Group, InnerEarLab, Department of Otolaryngology, University Medical Center, Göttingen, Germany

5 ENT-Department, County Hospital Kalmar, Kalmar, Sweden

6 Institute of Biomedicine, University of Aarhus, Aarhus, Denmark

7 MEMPHYS-Center for Biomembrane Physics, University of Southern Denmark, Odense, Denmark
8 Computational Biomolecular Dynamics Group, Max Planck Institute for Biophysical Chemistry, Göttingen, Germany

9 Pediatric Department, Aarhus University Hospital, Aarhus, Denmark

10 Maladies Sensorielles Genetiques, CHRU, Montpellier, France

11 INSERM U1051, Institute for Neurosciences of Montpellier, Montpellier, France

12 Universite Montpellier, Montpellier, France

13 Department of Otorhinolaryngology, Head and Neck Surgery and Audiology, Rigshospitalet/Gentofte Hospital, Hellerup, Denmark

14 Department of Audiology, Aarhus University Hospital, Aarhus, Denmark

15 Department of Ophthalmology, Aarhus University Hospital, Aarhus, Denmark

16 Eye Department Glostrup Hospital, Rigshospitalet, The Kennedy Centre, Glostrup, Denmark 
17 Department of Neurology, Aarhus University Hospital and University of Aarhus, Aarhus, Denmark

18 Audiological Research Centre, Faculty of Medicine and Health, Örebro University, Örebro, Sweden

19 Department of Neurotology, National Hospital for Neurology, Queen Square, London WC1N 3BG, UK

20 Department of Neurology, Great Ormond Street Hospital for Children NHS Foundation Trust, London WC1N 3JH, UK

21 North East Thames Regional Genetics Service, Great Ormond Street Hospital for Children NHS Foundation Trust, London WC1N 3JH, UK

22 Cochlear Implant Department, Great Ormond Street Hospital for Children NHS Foundation Trust, London WC1N 3JH, UK

23 Department of Audiovestibular Medicine, Great Ormond Street Hospital for Children NHS Foundation Trust, London WC1N 3JH, UK
24 Nuffield Hearing and Speech Centre, Royal National Throat Nose and Ear Hospital, London WC1X 8DA, UK

25 Genetic and Genomic Medicine Programme, UCL Great Ormond Street Institute of Child Health, London WC1N 1EH, UK

26 Institute of Human Genetics, Julius Maximilians University Würzburg, Würzburg, Germany

27 University Medical Centre, Institute of Human Genetics, Johannes Gutenberg University Mainz, Langenbeckstrasse 1, Mainz, Germany

28 Division of Pediatric Neurology, Department of Pediatric and Adolescent Medicine, University Medical Center, Göttingen, Germany

29 Institute for Auditory Neuroscience and InnerEarLab, University Medical Center, Göttingen, Germany 